\title{
$36 \mathrm{GHz}$ methanol lines from nearby galaxies: maser or quasi-thermal emission?`
}

\author{
P. K. Humire ${ }^{1}$, C. Henkel ${ }^{1,2,3}$, Y. Gong ${ }^{1}$, S. Leurini ${ }^{4,1}$, R. Mauersberger ${ }^{1}$, S. A. Levshakov ${ }^{5,6,7}$, B. Winkel ${ }^{1}$,
}

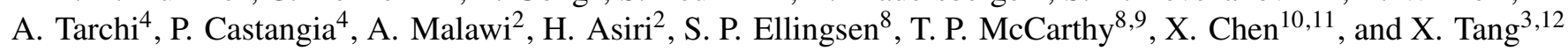

\author{
1 Max-Planck-Institut für Radioastronomie, Auf dem Hügel 69, 53121 Bonn, Germany \\ e-mail: chenkel@mpifr-bonn.mpg.de \\ 2 Astronomy Department, Faculty of Science, King Abdulaziz University, PO Box 80203, Jeddah 21589, Saudi Arabia \\ 3 Xinjiang Astronomical Observatory, Chinese Academy of Sciences, 830011 Urumqi, PR China \\ 4 INAF-Osservatorio Astronomico di Cagliari, Via della Scienza 5, 09047 Selargius, CA, Italy \\ 5 Ioffe Physical-Technical Institute, 194021 St. Petersburg, Russia \\ 6 Electrotechnical University "LETI”, 197376 St. Petersburg, Russia \\ 7 Petersburg Nuclear Physics Institute, 188300 Gatchina, Russia \\ 8 School of Natural Sciences, University of Tasmania, Hobart, TAS 7001, Australia \\ 9 Australia Telescope National Facility, CSIRO, PO Box 76, Epping, NSW 1710, Australia \\ 10 Center for Astrophysics, GuangZhou University, Guangzhou 510006, PR China \\ 11 Shanghai Astronomical Observatory, Chinese Academy of Sciences, Shanghai 200030, PR China \\ 12 Key Laboratory of Radio Astronomy, Chinese Academy of Sciences, 830011 Urumqi, PR China
}

Received 17 July 2019 / Accepted 12 November 2019

\begin{abstract}
Methanol $\left(\mathrm{CH}_{3} \mathrm{OH}\right)$ is one of the most abundant interstellar molecules, offering a vast number of transitions to be studied, including many maser lines. However, while the strongest Galactic $\mathrm{CH}_{3} \mathrm{OH}$ lines, the so-called class II masers, show no indications for the presence of superluminous counterparts in external galaxies, the less luminous Galactic class I sources appear to be different. Here we report class I $36 \mathrm{GHz}(\lambda \approx 0.8 \mathrm{~cm}) \mathrm{CH}_{3} \mathrm{OH} 4_{-1} \rightarrow 3_{0} \mathrm{E}$ line emission from the nearby galaxies Maffei $2(D \approx 6 \mathrm{Mpc})$ and IC 342 $(D \approx 3.5 \mathrm{Mpc})$, measured with the $100 \mathrm{~m}$ telescope at Effelsberg at three different epochs within a time span of about five weeks. The $36 \mathrm{GHz}$ methanol line of Maffei 2 is the second most luminous among the sources detected with certainty outside the Local Group of galaxies. This is not matched by the moderate infrared luminosity of Maffei 2 . Higher-resolution data are required to check whether this is related to its prominent bar and associated shocks. Upper limits for M 82, NGC 4388, NGC 5728 and Arp 220 are also presented. The previously reported detection of $36 \mathrm{GHz}$ maser emission in Arp 220 is not confirmed. Nondetections are reported from the related class I $44 \mathrm{GHz}(\lambda \approx 0.7 \mathrm{~cm})$ methanol transition towards Maffei 2 and IC 342, indicating that this line is not stronger than its $36 \mathrm{GHz}$ counterpart. In contrast to the previously detected $36 \mathrm{GHz} \mathrm{CH} 3 \mathrm{OH}$ emission in NGC 253 and NGC 4945 , our $36 \mathrm{GHz}$ profiles towards Maffei 2 and IC 342 are similar to those of previously detected nonmasing lines from other molecular species. However, by analogy to our Galactic center region, it may well be possible that the $36 \mathrm{GHz}$ methanol lines in Maffei 2 and IC 342 are composed of a large number of faint and narrow maser features that remain spatially unresolved. In view of this, a search for a weak broad $36 \mathrm{GHz}$ line component would also be desirable in NGC 253 and NGC 4945.
\end{abstract}

Key words. masers - galaxies: spiral - galaxies: individual: IC 342 - galaxies: individual: Maffei 2 - galaxies: ISM radio lines: galaxies

\section{Introduction}

Methanol $\left(\mathrm{CH}_{3} \mathrm{OH}\right)$ is one of the most abundant interstellar molecules (e.g., Kalenski \& Sobolev 1994; Wang et al. 2004; Maffucci et al. 2018) exhibiting a plethora of lines at centimeter $(\mathrm{cm})$, millimeter $(\mathrm{mm})$, and submillimeter (submm) wavelengths. To provide an example, Schilke et al. (2001) and Comito et al. (2005) reported the detection of a total of $\approx 650$ methanol transitions from Orion-KL. Some of these lines show inverted level populations and significant optical depths. Such methanol maser lines form two distinct families exhibiting clearly nonthermal emission in quite specific but different

* A copy of the reduced spectra is available at the CDS via anonymous ftp to cdsarc.u-strasbg.fr $(130.79 .128 .5)$ or via http: //cdsarc.u-strasbg.fr/viz-bin/cat/J/A+A/633/A106 transitions: class I masers are often separated from the main sources of star forming activity (between 0.1 and $1.0 \mathrm{pc}$ from UC HII regions, $\mathrm{OH}$ and $\mathrm{H}_{2} \mathrm{O}$ masers according to Gómez-Ruiz et al. 2016), whereas class II masers are closely associated with such sites. The $6.7 \mathrm{GHz}$ transition (Menten 1991) has become the prototypical class II maser line, exhibiting particularly strong emission in regions of intense radiation from warm dust and relatively cool gas (Cragg et al. 2005). Sources characterized by class I emission are encountered in regions not necessarily devoid of far infrared radiation but requiring collisional excitation - they appear to be associated with weak shocks, possibly related to outflows from young stellar objects interacting with the dense ambient interstellar medium (e.g., Kurtz et al. 2004; Leurini et al. 2016). In such regions, the $4_{-1} \rightarrow 3_{0} \mathrm{E}$ and $7_{0} \rightarrow 6_{1}$ $\mathrm{A}^{+}$transitions at 36 and $44 \mathrm{GHz}$, respectively, become most prominent. Apparently, the $36 \mathrm{GHz}$ line is strongest towards 


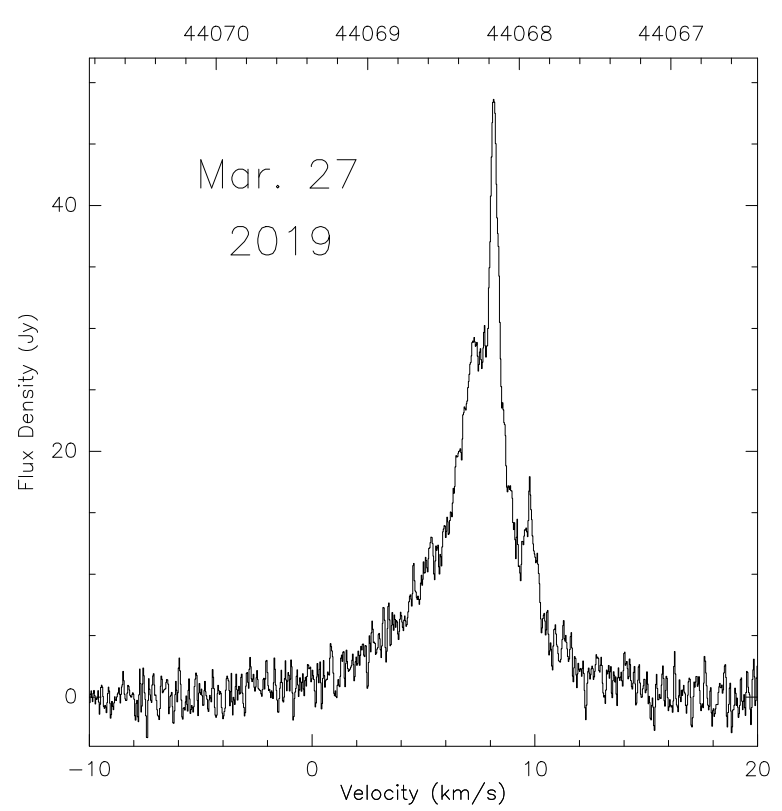

Fig. 1. $7_{0} \rightarrow 6_{1} \mathrm{~A}^{+}$methanol spectrum from Orion-KL. The adopted rest frequency is $44.069367 \mathrm{GHz}$, the beam size is $23^{\prime \prime}$ and the coordi-

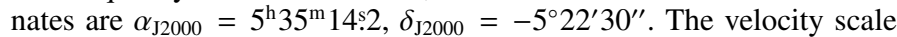
is the local standard of rest and the channel spacing is $0.31 \mathrm{~km} \mathrm{~s}^{-1}$ after averaging eight contiguous channels.

sources with kinetic temperatures of $T_{\text {kin }} \lesssim 100 \mathrm{~K}$, where signs of high-mass star formation are not yet seen. The $44 \mathrm{GHz}$ masers are closer to ultracompact (UC)-HII-regions and mm wave continuum sources and may be excited at $T_{\text {kin }} \gtrsim 100 \mathrm{~K}$. With respect to required densities, optimal conditions for $36 \mathrm{GHz}$ masers appear to include densities that are an order of magnitude higher than those at $44 \mathrm{GHz}$ (e.g., Pratap et al. 2008; McEwen et al. 2014; Nesterenok 2016; Leurini et al. 2016).

More than one thousand methanol masers are known in the Galaxy (e.g., Menten 1991; Pratap et al. 2008; Yusef-Zadeh et al. 2013; Cotton \& Yusef-Zadeh 2016; Yang et al. 2017, 2019). However, in extragalactic targets, such maser lines are rarely observed. Following the detections of quasi-thermal methanol emission in nearby galaxies (Henkel et al. 1987), the large number of Galactic masers and the existence of even more luminous $\mathrm{H}_{2} \mathrm{O}$ and $\mathrm{OH}$ "megamasers" (e.g., Lo 2005) provided strong motivation to search for class II $6.7 \mathrm{GHz}$ maser emission towards extragalactic sources (Ellingsen et al. 1994a; Phillips et al. 1998; Darling et al. 2003). This yielded detections in absorption towards NGC 3079 and Arp 220 (Impellizzeri et al. 2008; Salter et al. 2008) and in emission towards the Large Magellanic Cloud (LMC; Sinclair et al. 1992; Ellingsen et al. 1994b; Beasley et al. 1996; Green et al. 2008; Henkel et al. 2018a) and the Andromeda galaxy M 31 (Sjouwerman et al. 2010). The LMC also provided a $12.2 \mathrm{GHz}$ class II maser detection, reported by Ellingsen et al. (2010). However, the intrinsic brightness of all these emission lines turned out to be similar to those of their stronger Galactic counterparts.

Since none of the surveys for ultraluminous class II masers revealed positive results, searches for brighter analogs of the relatively inconspicuous Galactic class I masers were also carried out in nearby extragalactic sources. The detection of $36 \mathrm{GHz}$ class I masers in NGC 253 by Ellingsen et al. (2014) revealed emission that is more than ten times more luminous than the widespread emission associated with the $300 \mathrm{pc} \times 100 \mathrm{pc}$ central region of our Galaxy and $10^{4}$ times more luminous than a typical individual Galactic $36 \mathrm{GHz}$ maser. This demonstrates the existence of ultraluminous methanol masers (with respect to their Galactic counterparts). Follow-up observations confirmed this detection (Ellingsen et al. 2017; Gorski et al. 2017, 2019; Chen et al. 2018), which was augmented by successful searches for the related class I maser transitions at 44 and $84 \mathrm{GHz}$ in NGC 253 (Ellingsen et al. 2017; McCarthy et al. 2018a). Nevertheless, the search for additional sources turned out to be difficult. For some time, NGC 4945 was the only additional galaxy detected in the $36 \mathrm{GHz} \mathrm{CH}_{3} \mathrm{OH}$ class I maser line beyond any reasonable doubt (at multiple epochs with more than one telescope; see McCarthy et al. 2017, 2018b). More recently, $36 \mathrm{GHz}$ emission was also reported from IC 342 and NGC 6946 (Gorski et al. 2018). Chen \& Ellingsen (2018) very briefly mentioned a Jansky Very Long Baseline Array (JVLA) detection of Maffei 2 . In the following we report clear single-dish detections of Maffei 2, a particularly luminous emitter of $36 \mathrm{GHz}$ line radiation, and IC 342 in the $36.169261 \mathrm{GHz}$ (Endres et al. 2016) $4_{-1,4} \rightarrow 3_{0,3} \mathrm{E}$ transition of methanol, thus providing additional strong evidence for the widespread existence of such emission in nearby galaxies. This report also includes a number of nondetections, most notably the nondetection of Arp 220.

\section{Observations}

\subsection{Front- and backends}

The $36 \mathrm{GHz}$ measurements were carried out with the $100 \mathrm{~m}$ telescope at Effelsberg ${ }^{1}$ near Bonn, Germany, in early January and early February 2019. Complementary $44 \mathrm{GHz}$ observations were taken in late March 2019. We obtained the data with a secondary focus $Q$-band receiver, sensitive in the range $33-50 \mathrm{GHz}$, providing both circular polarizations with an equivalent system temperature of $\approx 130 \mathrm{Jy}$ on a flux density scale $(\approx 1.6 \mathrm{~K}$ main beam brightness temperature per Jy) prior to averaging the two polarizations. Pointing observations were carried out every $\approx 40$ min towards 3 C 84 and NRAO 150 near Maffei 2 and towards $\mathrm{W} 3(\mathrm{OH})$ near IC 342 . The pointing accuracy was $4^{\prime \prime} \pm 2^{\prime \prime}$ (the error is the standard deviation of an individual measurement) and never exceeded $10^{\prime \prime}$, while the beam size was $\approx 23^{\prime \prime}$ at both frequencies.

Near the start of each $36 \mathrm{GHz}$ observing session, we targeted the well-known $\mathrm{HC}_{3} \mathrm{~N} J=4 \rightarrow 3(36.392 \mathrm{GHz})$ and $\mathrm{SiS}$ $J=2 \rightarrow 1(36.310 \mathrm{GHz})$ lines of IRC+10216 to make sure the system was working. At $44.069 \mathrm{GHz}$, we chose the $\mathrm{CH}_{3} \mathrm{OH}$ $7_{0} \rightarrow 6_{1} \mathrm{~A}^{+}$line towards Orion-KL (Fig. 1) as a system check that may be used for future projects focusing on issues related to maser variability. For the measurements towards the targets themselves we used a position-switching mode, with 2 min offand on-source integration times, respectively, and with offsets in right ascension alternating between $+10^{\prime}$ and $-10^{\prime}$ to remove elevation-dependent effects. We employed a Fast Fourier Transform spectrometer backend with a bandwidth of $300 \mathrm{MHz}$. The number of channels was 65536, yielding a channel spacing of $0.038 \mathrm{~km} \mathrm{~s}^{-1}$ at $36 \mathrm{GHz}$ and a velocity resolution of $0.044 \mathrm{~km} \mathrm{~s}^{-1}$ (see Klein et al. 2012). At $44 \mathrm{GHz}$, the corresponding values are $0.031 \mathrm{~km} \mathrm{~s}^{-1}$ and $0.036 \mathrm{~km} \mathrm{~s}^{-1}$.

\subsection{Calibration}

We established flux density scales by performing continuum cross scans through 3C 286 and NGC 7027 (see Ott et al. 1994),

1 This publication is based on observations with the $100 \mathrm{~m}$ telescope of the MPIfR (Max-Planck-Institut für Radioastronomie) at Effelsberg. 

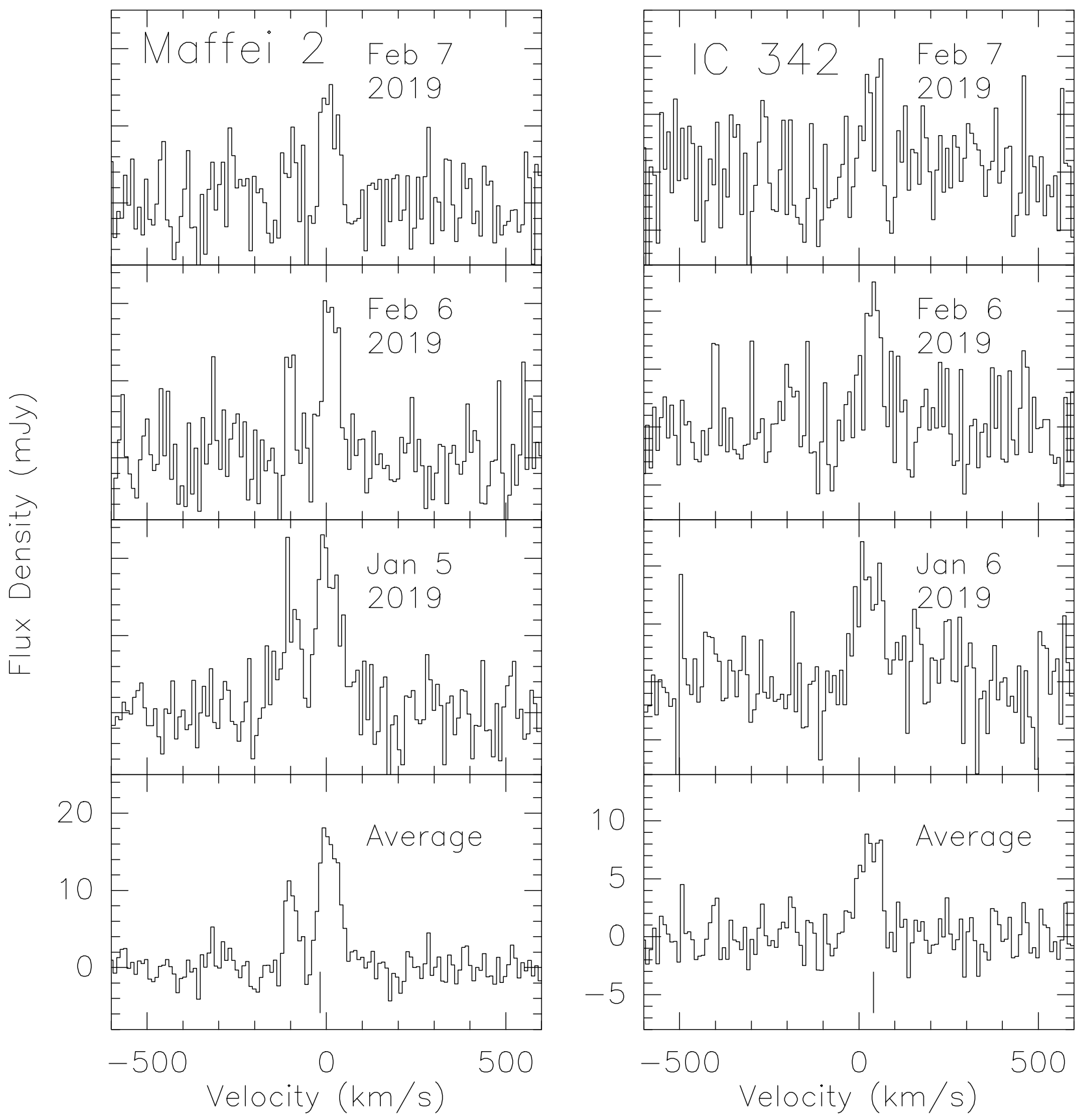

Fig. 2. $4_{-1} \rightarrow 3_{0}$ E methanol spectra from Maffei 2 (left) and IC 342 (right). Spectra from a given source are all presented with the same amplitude and velocity scale. The adopted rest frequency is $36.169261 \mathrm{GHz}$, the coordinates are $\alpha_{\mathrm{J} 2000}=2^{\mathrm{h}} 41^{\mathrm{m}} 5552, \delta_{\mathrm{J} 2000}=59^{\circ} 36^{\prime} 12^{\prime \prime}(\mathrm{Maffei} 2)$ and $\alpha_{\mathrm{J} 2000}=3^{\mathrm{h}} 46^{\mathrm{m}} 48 \mathrm{~s} 6, \delta_{\mathrm{J} 2000}=68^{\circ} 05^{\prime} 46^{\prime \prime}$ (IC 342). The velocity scale is the local standard of rest. The spectra were smoothed by averaging 256 contiguous channels to a channel spacing of $9.7 \mathrm{~km} \mathrm{~s}^{-1}$. The average spectrum was obtained, weighting individual subscans with the inverse square of their system temperature. The systemic local standard of rest velocity for Maffei 2 is $-14 \pm 5 \mathrm{~km} \mathrm{~s}^{-1}$ and $V_{\mathrm{HEL}}=V_{\mathrm{LSR}}-3.1 \mathrm{~km} \mathrm{~s}{ }^{-1}$. For IC 342 , the corresponding values are $+35 \pm 3 \mathrm{~km} \mathrm{~s}^{-1}$ and $V_{\mathrm{HEL}}=V_{\mathrm{LSR}}-3.9 \mathrm{~km} \mathrm{~s}^{-1}$. Vertical lines indicating the systemic velocities are included in the lower parts of the averaged spectra.

also accounting for gain variations of the telescope as a function of elevation and for a $0.6 \% \mathrm{yr}^{-1}$ secular decrease in intensity in the case of NGC 7027. Accounting for pointing errors and scatter in the measured signals, we estimate that the calibration uncertainty is $\pm 15 \%$.

\section{Results}

Figure 2 shows $36 \mathrm{GHz}$ methanol spectra taken towards the centers of Maffei 2 and IC 342. Following initial detections in early January 2019, which looked very promising but not fully convincing, we reobserved the sources a month later. With profiles from a total of three epochs, each showing signals above $3 \sigma$ and a total of order $10 \sigma$ (see Table 1 ), there is no doubt that the detections are real. However, we note that the lines obtained at the individual epochs are weak and relatively noisy. Therefore, differences with respect to peak flux density and line shape cannot be reliably interpreted in terms of variability. Instead, the differences are more likely caused by slightly varying average pointing offsets and, most likely, by different weather conditions, which included occasional light rain during the last observing night. This is consistent with the fact that the best single epoch 
Table 1. Methanol line parameters.

\begin{tabular}{|c|c|c|c|c|c|c|c|c|c|}
\hline Source & $\alpha_{\mathrm{J} 2000}$ & $\delta_{\mathrm{J} 2000}$ & Transition & $\begin{array}{c}v \\
(\mathrm{GHz})\end{array}$ & $\begin{array}{r}\int S \mathrm{~d} V \\
\left(\mathrm{mJy} \mathrm{km} \mathrm{s}^{-1}\right)\end{array}$ & $\begin{array}{r}V_{\mathrm{LSR}} \\
\left(\mathrm{km} \mathrm{s}^{-1}\right) \\
\end{array}$ & $\begin{array}{r}\Delta V_{1 / 2} \\
\left(\mathrm{~km} \mathrm{~s}^{-1}\right)\end{array}$ & $\begin{array}{l}S_{\text {peak }} \\
(\mathrm{mJy})\end{array}$ & $\begin{array}{c}L_{\mathrm{CH}_{3} \mathrm{OH}} \\
\left(L_{\odot}\right)\end{array}$ \\
\hline \multirow[t]{3}{*}{ Maffei 2} & $02: 41: 55.2$ & $+59: 36: 12$ & $4_{-1} \rightarrow 3_{0}$ & 36.169261 & $422 \pm 45$ & $-99.3 \pm 1.9$ & $35.0 \pm 3.9$ & $11.3 \pm 1.9$ & $0.53 \pm 0.06$ \\
\hline & & & $4_{-1} \rightarrow 3_{0}$ & 36.169261 & $1181 \pm 60$ & $7.5 \pm 1.5$ & $61.1 \pm 3.4$ & $18.1 \pm 1.5$ & $1.47 \pm 0.07$ \\
\hline & & & $7_{0} \rightarrow 6_{1}$ & 44.069419 & & & & $<12.0$ & \\
\hline \multirow[t]{2}{*}{ IC 342} & $03: 46: 48.6$ & $+68: 05: 46$ & $4_{-1} \rightarrow 3_{0}$ & 36.169261 & $641 \pm 64$ & $29.7 \pm 3.6$ & $70.6 \pm 8.0$ & $8.5 \pm 1.4$ & $0.26 \pm 0.03$ \\
\hline & & & $7_{0} \rightarrow 6_{1}$ & 44.069419 & & & & $<10.0$ & \\
\hline M 82 & $09: 55: 52.2$ & $+69: 40: 47$ & $4_{-1} \rightarrow 3_{0}$ & 36.169261 & & & & $<4.4$ & \\
\hline NGC 4388 & $12: 25: 46.7$ & $+12: 39: 41$ & $4_{-1} \rightarrow 3_{0}$ & 36.169261 & & & & $<4.6$ & \\
\hline NGC 5728 & $14: 42: 23.9$ & $-17: 15: 11$ & $4_{-1} \rightarrow 3_{0}$ & 36.169261 & & & & $<4.5$ & \\
\hline Arp 220 & $15: 34: 57.3$ & $+23: 30: 11$ & $4_{-1} \rightarrow 3_{0}$ & 36.169261 & & & & $<4.2$ & \\
\hline
\end{tabular}

Notes. Obtained from Gaussian fits to the average spectra displayed in Fig. 2. Column 1: sources; Cols. 2 and 3: J2000 coordinates; Col. 4: methanol transition; Col. 5: rest frequency; Cols. 6-8: integrated flux densities, local standard of rest velocities, and full width at half power line widths. Given errors include standard deviations from Gaussian fits but do not include the calibration uncertainty (Sect. 2.2); Col. 9: peak flux densities; Col. 10: methanol line luminosities. Adopted distances: 5.73 Mpc for Maffei 2 and 3.28 Mpc for IC 342 (see Sect. 4). For M 82, NGC 4388, NGC 5728, and Arp 220, $1 \sigma$ upper limits are given for $9.7 \mathrm{~km} \mathrm{~s}^{-1}$ wide channels. For the $44 \mathrm{GHz}$ transition towards Maffei 2 and IC $342,1 \sigma$ upper limits are given for $8.0 \mathrm{~km} \mathrm{~s}^{-1}$ wide channels. To convert the local standard of rest velocities given here into heliocentric values, use $V_{\mathrm{HEL}}=V_{\mathrm{LSR}}-3.1 \mathrm{~km} \mathrm{~s}^{-1}$ for Maffei 2 and $V_{\mathrm{HEL}}=V_{\mathrm{LSR}}-3.9 \mathrm{~km} \mathrm{~s}^{-1}$ for IC 342 .

spectra are from January 2019, while the latest spectra show the lowest signal-to-noise ratios.

Table 1 provides Gaussian fit parameters to the average spectra from Maffei 2 and IC 342. In Maffei 2 we find two well-separated spectral components, while IC 342 exhibits one component and overall weaker emission, with lower peak and velocity integrated flux density. The $\mathrm{CH}_{3} \mathrm{OH}$ line luminosities in the last column of Table 1, assuming isotropic radiation, were calculated using

$$
L / L_{\odot}=0.038 \times\left(S_{v} \Delta V_{1 / 2} / \mathrm{Jy} \mathrm{km} \mathrm{s}^{-1}\right) \times(D / \mathrm{Mpc})^{2},
$$

with $S_{v}, \Delta V_{1 / 2}$ and $D$ representing flux density, full width to half power line width and distance. Upper limits are provided for four additional galaxies as well as also for the $44 \mathrm{GHz}$ line of methanol towards Maffei 2 and IC 342.

\section{Discussion}

\subsection{Detected lines}

The two originally detected $36 \mathrm{GHz}$ galaxies outside the Local Group, NGC 253 and NGC 4945 (Ellingsen et al. 2014; McCarthy et al. 2017) show overall properties that are quite different from the galaxies detected by us. Both NGC 253 and NGC 4945 host ongoing starbursts and are characterized by infrared luminosities a few times higher than those of the Milky Way, Maffei 2 or IC 342. NGC 4945 also hosts an active galactic nucleus. NGC 253 and NGC 4945 are both highly inclined giant spiral galaxies $\left(i \approx 75^{\circ}\right.$; e.g., Iodice et al. 2014; Henkel et al. 2018b). Furthermore, with outflowing gas in their nuclear regions (Turner 1985; Bolatto et al. 2013; Henkel et al. 2018b), they also provide an environment in which shocks potentially leading to class I methanol maser emission may be ubiquitous. It is remarkable, that the various maser components in NGC 253 occur not right at the center but at offsets of a few hundred parsecs from the nucleus (Ellingsen et al. 2017). The same also holds in the case of NGC 4945, where the (southeastern) front side of the galaxy shows a single but relatively strong maser spot, presumably also arising at a distance of a few hundred parsecs from the nucleus (McCarthy et al. 2018b).

Maffei 2 and IC 342, located behind the Galactic plane, are different. To evaluate their global properties, the first notable parameter is distance. While IC 342 is located at about the same distance as NGC 253 and NGC 4945 (here we adopt $3.28 \mathrm{Mpc}$; Karachentsev et al. 2013), Maffei 2 may be located at a significantly greater distance. Wu et al. (2014) estimated distances of 3.4-3.5 Mpc for both Maffei 2 and IC 342 using the tip of the red giant branch method. However, Tikhonov \& Galazutdinova (2018) re-evaluated their Hubble Space Telescope (HST) images. They applied stellar photometry at infrared and optical spectral ranges and compared the resulting Hertzprung-Russel diagrams. In particular the red giant branches, with their upper luminosity boundaries and color indices, were analyzed and compared with data from galaxies being much less obscured by Galactic dust. These latter authors obtained extinctions of $A_{\mathrm{v}}=3 \mathrm{~m} .65$ and 0.9 for Maffei 2 and IC 342, respectively. While their resulting distance to IC 342 is consistent with previously obtained values, their distance to Maffei 2 becomes $6.7 \pm 0.5 \mathrm{Mpc}$. Reanalyzing near infrared photometry from the HST, Anand et al. (2019) confirm a larger distance for Maffei 2 and propose $D=5.73 \pm 0.40 \mathrm{Mpc}$, which is adopted in the following (see also Table 1 for both Maffei 2 and IC 342).

A common feature of Maffei 2 and IC 342 is, as already briefly mentioned, their location behind the plane of the Milky Way. While IC 342 is displaced from the plane by 10 :6, Maffei 2 is almost exactly on this plane ( $\left.b^{\mathrm{II}}=-0.3\right)$, which led to its very late discovery (Maffei 1968). With respect to infrared luminosity and therefore also to star formation, making use of the more recent distance estimates to Maffei 2, both galaxies have luminosities of $L_{\mathrm{IR}} \approx 10^{10} L_{\odot}$. Not hosting an AGN, they are about as active as our own Galaxy $\left(L_{\mathrm{FIR}} \approx 1.2 \times 10^{10} L_{\odot}\right.$; e.g., Misiriotis et al. 2004, 2006), forming on average a few solar masses of new stars per year (see, e.g., Kennicutt 1998 for the correlation between infrared luminosity and star formation rate and Chomiuk \& Povich 2011 for uncertainties related to estimates of Galactic star formation rates). Both galaxies host two spiral arms terminating on a central ring with vigorous massive star formation (e.g., Meier \& Turner 2012). Therefore, chemically, Maffei 2 may be considered to some extent as a more inclined copy of IC 342 and may not (as before) be considered as a particularly small spiral galaxy. However, one major difference remains: it is the kiloparsec-sized bar in Maffei 2 that is possibly causing widespread shocks favoring $36 \mathrm{GHz}$ 
methanol maser emission (see Ellingsen et al. 2017 and below). A similar structure is not found in IC 342.

The previously obtained $36 \mathrm{GHz}$ methanol maser detections in NGC 253 and NGC 4945 (Ellingsen et al. 2014; McCarthy et al. 2017) have something in common: the detected profiles are much narrower than those obtained when measuring quasithermal line emission from areas covering their entire central molecular zones. This is different with respect to our lines shown in Fig. 2. Galactic foreground emission (Maffei 2 and IC 342 are located behind the Perseus arm) cannot cause this effect. The two line peaks detected towards Maffei 2 are not compatible with molecular velocities of the Perseus arm (Cohen et al. 1980) and must therefore be of extragalactic origin. The $36 \mathrm{GHz}$ methanol velocity components are also seen, with similar spectral profiles but with lower signal-to-noise ratios, in other tracers of dense molecular gas, the integrated ammonia $\left(\mathrm{NH}_{3}\right)(J, K)=(1,1)$ and (likely) (2,2) profiles near $24 \mathrm{GHz}$ (Lebrón et al. 2011). The global ammonia profiles from IC 342 also show similar shapes and radial velocities to those presented in Fig. 2 and cannot be interpreted in terms of Galactic origin, since Galactic molecular clouds in this region show smaller radial velocities (Cohen et al. 1980; Lo et al. 1984; Tarchi et al. 2002). Both $36 \mathrm{GHz}$ methanol (e.g., Leurini et al. 2016) and $24 \mathrm{GHz}$ ammonia (e.g., Lebrón et al. 2011) may trace similarly dense molecular gas because rates for spontaneous emission are not far apart $\left(1.48\right.$ and $1.67 \times 10^{-7} \mathrm{~s}^{-1}$, the latter for the $(J, K)=(1,1)$ ammonia transition; e.g., Schöier et al. 2005 and references therein). Therefore, the agreement in line shapes does not directly hint at $36 \mathrm{GHz}$ maser emission in our two detected galaxies, but this is further discussed below in relation to what is known from the central region of the Milky Way.

Following the nomenclature of Lebrón et al. (2011), used for their ammonia $\left(\mathrm{NH}_{3}\right)$ observations with a beam size of $\approx 3^{\prime \prime} .5$, the blueshifted component we recognize in Maffei 2 (Fig. 2 and Table 1) must arise from their regions $C$ and $D$, located in the southern part of the galaxy. The component near zero velocity is related to the northern part, represented by molecular complexes A and B. In Fig. 2 of Lebrón et al. (2011), sources B and C are dominant and, with their separation of $10^{\prime \prime}-15^{\prime \prime}(280-415 \mathrm{pc})$, they should also dominate the $36 \mathrm{GHz}$ methanol emission inside our $23^{\prime \prime}$ beam. In the para- $\mathrm{NH}_{3}(1,1)$ ground state inversion transition, the redshifted component is stronger than the blueshifted one, by a factor of roughly 1.6. The corresponding line intensity ratio we see in our $36 \mathrm{GHz}$ methanol transition is $2.8 \pm 0.3$ in favor of this more redshifted component. While this difference is clearly compatible with quasi-thermal emission in both $\mathrm{NH}_{3}$ (where this is expected) and $\mathrm{CH}_{3} \mathrm{OH}$, although suggesting slightly different physical or chemical conditions, we nevertheless note that the blueshifted $\mathrm{CH}_{3} \mathrm{OH}$ component may be narrower than the one seen in ammonia.

Toward IC 342, our line widths and velocities of methanol are comparable, within the errors, to the ammonia lines from Lebrón et al. (2011). Very recently, Gorski et al. (2018) reported JVLA measurements of the central kiloparsec of IC 342 with $1^{\prime \prime}$ and $7 \mathrm{~km} \mathrm{~s}^{-1}$ resolution. These latter authors detected six spatially resolved $36 \mathrm{GHz} \mathrm{CH}_{3} \mathrm{OH}$ sites in IC 342 located along a northeast-southwest axis (position angle $\approx 30^{\circ}$ ) with a length of $20^{\prime \prime}(300 \mathrm{pc})$. Interestingly, their strongest four sources peak at velocities clearly redshifted $\left(\approx 50 \mathrm{~km} \mathrm{~s}^{-1}\right)$ with respect to the galaxy's systemic local standard of rest velocity of $35 \mathrm{~km} \mathrm{~s}^{-1}$. A similar trend is also seen in the $\mathrm{NH}_{3}(1,1)$ emission from Lebrón et al. (2011; their Fig. 1, lower right panel). However, our Table 1 instead indicates a peak velocity of the overall emission at systemic or slightly blueshifted velocities. Since the Gorski et al.
(2018) data encompass the entire central region of IC 342 giving rise to emission from high-density molecular tracers, this discrepancy cannot be explained in terms of spatial regions not being covered by their measurements. This also holds for our data. Missing flux in the interferometric measurements might instead be the cause of the difference. However, adding the luminosities given in their Table 6 leads to a total luminosity of $0.315 L_{\odot}$, which is (within $2 \sigma$, see, e.g., Sect. 2.2) compatible with our luminosity of $(0.26 \pm 0.03) L_{\odot}$ (Table 1$)$. While we cannot explain the difference in radial velocities, the apparent lack of missing flux suggests that the bulk of the $36 \mathrm{GHz}$ methanol emission is originating from compact spatially unresolved regions, compatible with maser emission.

In the following text we use the definition of starburst galaxies as outlined by Mao et al. (2010; their Fig. 1), where the optical isophotal diameter $D_{25}\left(25^{\mathrm{m}} / \mathrm{arcsec}^{2}\right)$ is applied to obtain the ratio $\log \left[\left(L_{\mathrm{IR}} / L_{\odot}\right) /\left(D_{25}^{2} / \mathrm{kpc}^{2}\right)\right]$. Here, in almost all cases the value 7.25 separates galaxies commonly identified as starburst from nonstarburst galaxies. With Maffei 2, IC 342 and NGC 6946 not being starburst galaxies like NGC 253 and NGC 4945 (according to our definition), but showing infrared luminosities and likely star forming rates similar to those of the Milky Way, there may be similarities between the conditions in Maffei 2, IC 342, and NGC 6946 and the central molecular zone (CMZ) of our Galaxy. Haschick et al. (1990) provided data from our Galactic center region, showing $36 \mathrm{GHz}$ and $44 \mathrm{GHz}$ methanol spectra from $\mathrm{Sgr} \mathrm{A}-\mathrm{A} \approx 1^{\prime}$ northeast of the Galactic center and Sgr A-F, $\approx 3^{\prime}$ south of the Galactic center (their Fig. 9). These latter authors find narrow $36 \mathrm{GHz}$ spikes, likely due to maser emission, on top of a broad component, possibly of quasi-thermal origin. For these two Galactic sources, the $36 \mathrm{GHz}$ methanol lines show quite a number of narrow spikes, while only one such spike is seen in the corresponding $44 \mathrm{GHz}$ methanol line.

More recently, Yusef-Zadeh et al. (2013) and Cotton \& Yusef-Zadeh (2016) find a widespread population of more than 2000 compact $36 \mathrm{GHz}$ methanol maser sources within the central degree of our Galaxy. The lines are narrow $\left(\approx 1 \mathrm{~km} \mathrm{~s}^{-1}\right)$. Such a widespread distribution with a huge number of individual maser components covering the full radial velocity range of the nuclear environment could mimic a total profile, that is quite undistinguishable from quasi-thermal emission as long as the masers are spatially unresolved and no individual source stands out.

Figure 3 correlates $36 \mathrm{GHz}$ line methanol luminosities with infrared luminosities obtained from IRAS (the Infrared Astronomical Satellite) and therefore with the star forming rates of their respective parent galaxies. While the number of sources is small and while both $36 \mathrm{GHz}$ line and infrared continuum luminosities may be uncertain, it is clear that there is no good correlation. NGC 253 with its high infrared luminosity shows the most luminous $36 \mathrm{GHz}$ methanol emission. However, NGC 4945, almost as luminous in the infrared, is comparatively weak with respect to the $36 \mathrm{GHz}$ methanol line. Outflowing gas in its nuclear region (Henkel et al. 2018b), leading to shocks, is possibly not quite as prominent as in NGC 253 (Turner 1985; Bolatto et al. 2013). Maffei 2 also stands out with respect to methanol emission, even though its infrared luminosity is modest. While we did not resolve the sources, the explanation may be the prominent nuclear bar in Maffei 2 (e.g., Meier \& Turner 2012), that may cause shocks throughout its central region, enhancing $36 \mathrm{GHz}$ methanol emission above its normal level. Higher-resolution observations to study the spatial connection between the bar and the $36 \mathrm{GHz}$ methanol emission would be desirable. 


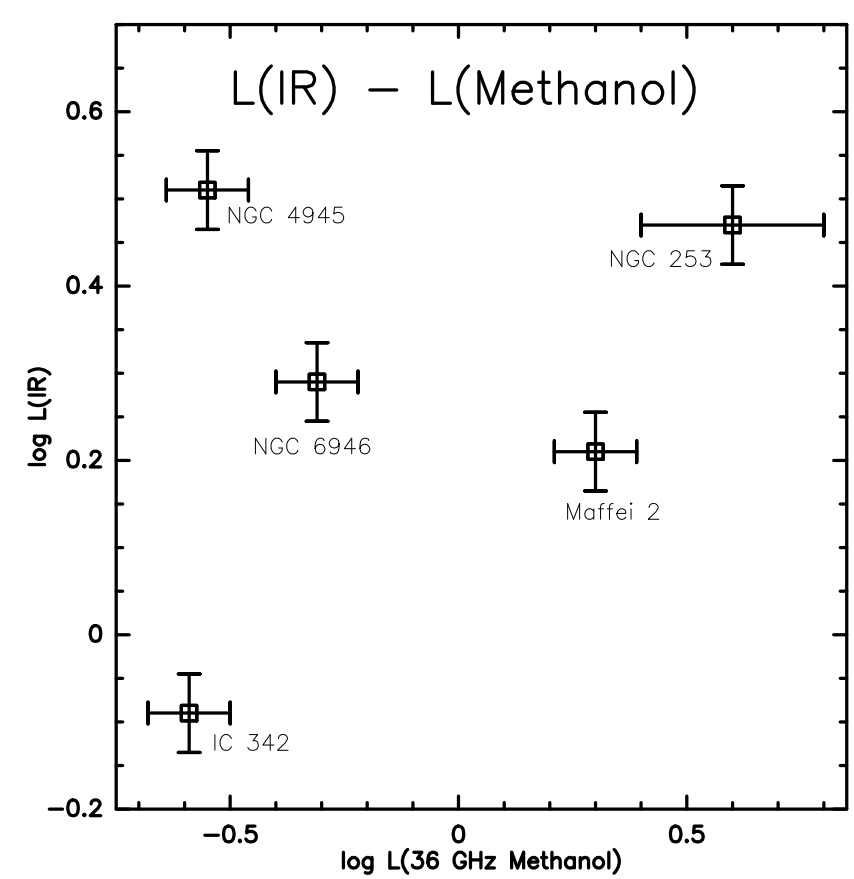

Fig. 3. $36 \mathrm{GHz}$ methanol line luminosities in solar units vs. infrared luminosities in units of $10^{10} L_{\odot}$, obtained from IRAS data, on logarithmic scales. For the former, $\pm 20 \%$ (see Sect. 2.2, we added $5 \%$ due to limited signal-to-noise ratios) and for the latter $\pm 10 \%$ error bars have been taken. The exception is NGC 253, where a larger error bar has been chosen for the $36 \mathrm{GHz}$ methanol luminosity (see below). Adopted distances to the galaxies are $3.28 \mathrm{Mpc}$ for IC 342 (Sect. 4), $5.73 \mathrm{Mpc}$ for Maffei 2 (Sect.4), 5.98 Mpc for NGC 6946 (Gorski et al. 2018), 3.5 Mpc for NGC 253 (Gorski et al. 2018) and 3.8 Mpc for NGC 4945 (Henkel et al. 2018b). The methanol line luminosities were calculated with Eq. (1). There exist huge discrepancies in total $36 \mathrm{GHz}$ methanol flux density in the case of NGC 253, with Ellingsen et al. (2014) finding $\approx 0.3 L_{\odot}$, Ellingsen et al. (2017) reporting $\approx 3 L_{\odot}$ and Gorski et al. (2017) suggesting $6.4 L_{\odot}$. We chose $4 L_{\odot}$. For NGC 4945 , McCarthy et al. (2017) report $0.14 L_{\odot}$, while McCarthy et al. (2018b) find $2.8 L_{\odot}$. We have taken the latter value, suspecting that the lower luminosity values are due to missing flux (e.g., Chen et al. 2018) and that variability might also play a role.

As already mentioned, it is noteworthy that toward the nonstarbursting galaxies we see line profiles compatible with the line profiles of other dense molecular gas tracers (for $\mathrm{NH}_{3}$, see the discussion above; for HNCO, see Gorski et al. 2017, 2018). Toward the two detected starburst galaxies, however, we see emission from highly confined regions, the strongest such region being located in NGC 4945. We may therefore ask whether these galaxies also contain a component similar to the one in the less active galaxies Maffei 2, IC 342, and NGC 6946, only being less conspicuous in view of the outstanding maser peaks already encountered in these starburst environments. We advocate searches for such an underlying smoother $36 \mathrm{GHz}$ emission component also in these galaxies. We note however that by analogy with our Galactic center region (e.g., Yusef-Zadeh et al. 2013), it is likely that this component is also representing maser emission.

\subsection{Undetected lines}

It may be argued that our lack of detected $44 \mathrm{GHz}$ methanol in Maffei 2 and IC 342 is caused by the different levels of excitation, with the $36 \mathrm{GHz}$ upper level $\left(4_{-1}\right) 21 \mathrm{~K}$ and the $44 \mathrm{GHz}$ upper level $\left(7_{0}\right) 65 \mathrm{~K}$ above the ground state (the equiv- alent energy of the photons is only $\approx 2 \mathrm{~K}$ ). However, we note that our $44 \mathrm{GHz}$ measurements show rms values that are about three times larger than those at $36 \mathrm{GHz}$. Therefore, our nondetections at $44 \mathrm{GHz}$ toward Maffei 2 and IC 342 merely indicate that the $44 \mathrm{GHz}$ emission is not stronger than that at $36 \mathrm{GHz}$, which is consistent with the conditions in NGC 253 (Ellingsen et al. 2017) and appears to be a typical signature of extragalactic methanol class I emission.

With respect to our $36 \mathrm{GHz}$ nondetections, the Arp 220 result is particularly interesting. Chen et al. (2015) reported several areas with emission in the outskirts of the merger with flux densities of order $10-20 \mathrm{mJy}$ and our beam size is large enough to incorporate most of these regions. However, our upper limit given in Table 1 (last line) corresponds to a $5 \sigma$ level of $\approx 4 \mathrm{mJy}$ for a feature of $300 \mathrm{~km} \mathrm{~s}^{-1}$ in width. In view of its extreme infrared flux density, M 82 was an obvious target (it is one of the most prolific extragalactic infrared emitters of the entire sky; e.g., Henkel et al. 1986). Instead, NGC 4388 and NGC 5728 were observed because Chen et al. (2016) had published tentative $36 \mathrm{GHz}$ class I methanol signals. We were not able to confirm their tentative features but note that our data are of similar sensitivity, and so in these cases, unlike for Arp 220, the situation remains unclear.

\section{Conclusions}

With Maffei 2 and IC 342 we present two $36 \mathrm{GHz}$ class I methanol line emitters from galaxies outside the Local Group, while a previously reported detection of the ultraluminous infrared galaxy Arp 220 is not confirmed. The line luminosity of Maffei 2 is surprisingly high, which is possibly related to its prominent bar. As in the prototypical source, NGC 253, upper limits indicate that the $44 \mathrm{GHz}$ class I methanol line is not substantially stronger. Now there are five such well-confirmed sources with $36 \mathrm{GHz}$ line detections beyond any reasonable doubt. In contrast to the two initially detected and still most prominent of these sources, NGC 253 and NGC 4945, it is difficult to directly prove that the detected signals from Maffei 2 and IC 342 (and NGC 6946) are caused by masers. The line profiles are compatible with those obtained from ammonia, where masers are rarely observed. Unlike NGC 253 and NGC 4945, which are starburst galaxies, the others are spiral galaxies that are about as active with respect to star formation as our Milky Way. Therefore, by analogy with the central part of our Galaxy, the most likely interpretation is that the detected features represent the superposition of a large number of spatially unresolved weak maser hotspots lacking a single dominant source. It is hard to imagine that more active galaxies, like for example NGC 253 and NGC 4945, do not also show such a population of weak masers. Therefore, a search for such a weak and broad underlying spatially widespread $36 \mathrm{GHz}$ methanol emission would also be worthwhile in starburst galaxies, as well as interferometric studies with utmost sensitivity to unambiguously demonstrate that at least a part of the detected emission originates from maser sources. The latter would, however, be a very ambitious project, presumably only feasible with the future next generation Very Large Array (ngVLA).

Acknowledgements. We wish to thank K.-M. Menten and an anonymous referee for valuable comments as well as the staff at Effelsberg for their great support, in particular the operators and A. Kraus. S. A. L. acknowledges funding through RSF grant No. 19-12-00157. This research has made use of NASA's Astrophysical Data System and of the NASA/IPAC Extragalactic Database. 


\section{References}

Anand, G. S., Tully, R. B., Rizzi, L., \& Karachentsev, I. D. 2019, ApJ, 872, 14 Beasley, A. J., Ellingsen, S. P., Claussen, M. J., \& Wilcots, E. 1996, ApJ, 459, 600

Bolatto, A. D., Warren, S. R., Leroy, A. K., et al. 2013, Nature, 499, 450

Chen, X., \& Ellingsen, S. P. 2018, in Astrophysical Masers: Unlocking the Mysteries of the Universe, eds. A. Tarchi, M. J. Reid, \& P. Castangia, IAU Symp., 336, 99

Chen, X., Ellingsen, S. P., Baan, W. A., et al. 2015, ApJ, 800, L2

Chen, X., Ellingsen, S.-P., Zhang, J.-S., et al. 2016, MNRAS, 459, 357

Chen, X., Ellingsen, S. P., Shen, Z.-Q., et al. 2018, ApJ, 856, L35

Chomiuk, L., \& Povich, M. S. 2011, AJ, 142, 197

Cohen, R. S., Cong, H., Dame, T. M., \& Thaddeus, P. 1980, ApJ, 239, L53

Comito, C., Schilke, P., Phillips, T. G., et al. 2005, ApJS, 156, 127

Cotton, W. D., \& Yusef-Zadeh, F. 2016, ApJS, 227, 10

Cragg, D. M., Sobolev, A. M., \& Godfrey, P. D. 2005, MNRAS, 360, 533

Darling, J., Goldsmith, P., Li, D., \& Giovanelli, R. 2003, AJ, 125, 1177

Ellingsen, S. P., Norris, R. P., Whiteoak, J. B., et al. 1994a, MNRAS, 267, 510

Ellingsen, S. P., Whiteoak, J. B., Norris, R. P., Caswell, J. L., \& Vaile, R. A. 1994b, MNRAS, 269, 1019

Ellingsen, S. P., Breen, S. L., Caswell, J. L., Quinn, L. J., \& Fuller, G. A. 2010 , MNRAS, 404, 779

Ellingsen, S. P., Chen, X., Qiao, H.-H., et al. 2014, ApJ, 790, L28

Ellingsen, S. P., Chen, X., Breen, S. L., \& Qiao, H.-H. 2017, MNRAS, 472, 604

Endres, C. P., Schlemmer, S., Schilke, P., Stutzki, J., \& Müller, H. S. P. 2016, J. Mol. Spectr., 327, 95

Gómez-Ruiz, A. I., Kurtz, S. E., Araya, E. D., Hofner, P., \& Loinard, L. 2016 ApJS, 222, 18

Gorski, M., Ott, J., Rand, R., et al. 2017, ApJ, 842, 124

Gorski, M., Ott, J., Rand, R., et al. 2018, ApJ, 856, 134

Gorski, M., Ott, J., Rand, R., et al. 2019, MNRAS, 483, 5434

Green, J. A., Caswell, J. L., Fuller, G. A., et al. 2008, MNRAS, 385, 948

Haschick, A. D., Menten, K. M., \& Baan, W. A. 1990, ApJ, 354, 556

Henkel, C., Wouterloot, J. G. A., \& Bally, J. 1986, A\&A, 155, 193

Henkel, C., Jacq, T., Mauersberger, R., Menten, K. M., \& Steppe, H. 1987, A\&A 188, L1

Henkel, C., Greene, J. E., \& Kamali, F. 2018a, in Astrophysical Masers: Unlocking the Mysteries of the Universe, eds. A. Tarchi, M. J. Reid, \& P. Castangia, IAU Symp., 336, 69

Henkel, C., Mühle, S., Bendo, G., et al. 2018b, A\&A, 615, A155

Impellizzeri, C. M. V., Henkel, C., Roy, A. L., \& Menten, K. M. 2008, A\&A, 484, L43

Iodice, E., Arnaboldi, M., Rejkuba, M., et al. 2014, A\&A, 567, A86

Kalenski, S. V., \& Sobolev, A. M. 1994, Astron. Lett., 20, 91

Karachentsev, I. D., Makarov, D. I., \& Kaisina, E. I. 2013, AJ, 145, 101
Kennicutt, R. C. 1998, ARA\&A, 36, 189

Klein, B., Hochgürtel, S., Krämer, I., et al. 2012, A\&A, 542, L3

Kurtz, S., Hofner, P., \& Álvarez, C. V. 2004, ApJS, 155, 149

Lebrón, M., Mangum, J. G., Mauersberger, R., et al. 2011, A\&A, 534, A56

Leurini, S., Menten, K. M., \& Walmsley, C. M. 2016, A\&A, 592, A31

Lo, K. Y. 2005, ARA\&A, 43, 625

Lo, K. Y., Berge, G. L., Claussen, M. J., et al. 1984, ApJ, 282, L59

Maffei, P. 1968, PASP, 80, 618

Maffucci, D. M., Wenger, T. V., Le Gal, R., \& Herbst, E. 2018, ApJ, 868, 41

Mao, R.-Q., Schulz, A., Henkel, C., et al. 2010, ApJ, 724, 1336

McCarthy, T. P., Ellingsen, S. P., Chen, X., et al. 2017, ApJ, 846, 156

McCarthy, T. P., Ellingsen, S. P., Breen, S. L., Voronkov, M. A., \& Chen, X. 2018a, ApJ, 867, L4

McCarthy, T. P., Ellingsen, S. P., Breen, S. L., et al. 2018b, MNRAS, 480, 4578

McEwen, B. C., Pihlström, Y. M., \& Sjouwerman, L. O. 2014, ApJ, 793, 133

Meier, D. S., \& Turner, J. L. 2012, ApJ, 755, 104

Menten, K. M. 1991, ApJ, 380, L75

Misiriotis, A., Papadakis, I. E., Kylafis, N. D., \& Papamastorakis, J. 2004, A\&A, 417, 39

Misiriotis, A., Xilouris, E. M., Papamastorakis, J., Boumis, P., \& Goudis, C. D. 2006, A\&A, 459, 113

Nesterenok, A. V. 2016, MNRAS, 455, 3978

Ott, M., Witzel, A., Quirrenbach, A., et al. 1994, A\&A, 284, 331

Phillips, C. J., Norris, R. P., Ellingsen, S. P., \& Rayner, D. P. 1998, MNRAS, 294, 265

Pratap, P., Shute, P. A., Keane, T. C., Battersby, C., \& Sterling, S. 2008, AJ, 135, 1718

Salter, C., Ghosh, T., Catinella, B., et al. 2008, AJ, 136, 389

Schilke, P., Benford, D. J., Hunter, T. R., Lis, D. C., \& Phillips, T. G. 2001, ApJS, 132,281

Schöier, F. L., van der Tak, F. F. S., van Dishoeck, E. F., \& Black, J. H. 2005, A\&A, 432, 369

Sinclair, M. W., Carrad, G. J., Caswell, J. L., Norris, R. P., \& Whiteoak, J. B. 1992, MNRAS, 256, 33

Sjouwerman, L. O., Murray, C. E., Pihlström, Y. M., Fish, V. L., \& Araya, E. D. 2010, ApJ, 724, L158

Tarchi, A., Henkel, C., Peck, A. B., \& Menten, K. M. 2002, A\&A, 385, 1049

Tikhonov, N. A., \& Galazutdinova, O. A. 2018, Astrophys. Bull., 73, 279

Turner, B. E. 1985, ApJ, 299, 312

Wang, M., Henkel, C., Chin, Y.-N., et al. 2004, A\&A, 422, 883

Wu, P. F., Tully, R. B., \& Rizzi, L. 2014, AJ,148, 7

Yang, K., Chen, X., Shen, Z.-Q., et al. 2017, ApJ, 846, 160

Yang, K., Chen, X., Shen, Z.-Q., et al. 2019, ApJS, 241, 18

Yusef-Zadeh, F., Cotton, W., Viti, S., Wardle, M., \& Royster, M. 2013, ApJ, 764, L19 